\title{
JAMES WADDELL ALEXANDER 1888-1971
}

\section{BY LEON W. COHEN}

In 1922 there appeared $A$ proof and extension of the Jordan-Brouwer separation theorem by $\mathrm{J}$. W. Alexander. This work was a major influence in the flowering of algebraic topology which occurred in the ensuing fifty years. It contained a duality theorem algebraically similar to, but geometrically different from, the classical Poincaré duality theorem. In establishing the equality of the $i$ th Betti number of a complex embedded topologically in an $n$-sphere and the $(n-i-1)$ st Betti number of its complement, Alexander indicated in this paper how homology theory could arise in open and closed sets. This set the stage for algebraic topology in metric space, a line of investigation vigorously pursued by Alexandroff, Čech, Vietoris, and Wilder.

There were far reaching algebraic consequences. The theorems of Alexander and Poincaré essentially asserted the isomorphism of pairs of finitely generated abelian groups, the groups in each pair reflecting geometric properties in dual dimensions. This started a search for a purely algebraic theorem which embodied the group theoretic relations inherent in the geometrical settings of the two duality theorems. The relevant result was found by Pontrjagin (cf. L. Pontrjagin, Über den algebraischen Inhalt topologischer Dualitätssätze, Math. Ann. 105 (1931).) The association of Betti groups with open and closed sets leads to the problem of classifying abelian groups with countably many generators. Alexander dealt with this problem during 1931-1935, and found an appropriate decomposition of such groups into direct summands. In this investigation Alexander initiated the application of topological groups to algebraic topology (cf. L. Pontrjagin, The general topological theorem of duality for closed sets, Ann. of Math. 35 (1934).)

Alexander went on to find a role for ring theory in topology. Earlier Lefschetz had studied intersections of geometrical $i$-chains and $(n-i)$ chains on $n$-manifolds, exploiting Poincaré's theorem. Alexander considered the linking of geometrical $i$-cycles on a complex in an $n$-sphere with $(n-i-1)$-cycles in its complement. It turned out that in both cases multiplication of the algebraic counterparts of these geometrical entities was the appropriate technique, and that the homology groups could be embedded in rings which yielded new invariants. This work of Alexander was simultaneous with the same results obtained by Kolmogorov, both men reporting their results at the First International Topological Conference in Moscow, 1935. These beginnings were further developed by 
Whitney (cf. Hassler Whitney, On products in a complex, Ann. of Math. 39 (1938). It contains an illuminating historical note on p. 398), and pointed the way to cohomology theory which is algebraically dual to the theory of homology.

Alexander extended his duality theorem to a class of sets in Hilbert space. His last works included the application of his connectivity ring to abstract spaces and to topology in a lattice. Besides work on his major themes Alexander produced a fixed point theorem, a topological 2-sphere whose complement in 3-space is not simply connected, contributions to the theory of knots, and, in an early paper (1915), a theorem on univalent functions which is still being quoted.

Alexander's mathematical life was lived in Princeton where he carried on the topological tradition established by Veblen. He was a student at the university, receiving its B.S. (1910), A.M. (1911), and Ph.D. (1915). From 1911 to 1933 Alexander was a member of the Princeton faculty, resigning to accept a professorship in The Institute for Advanced Study. He was elected to the National Academy of Sciences, Washington, in 1930. In 1947 the universities in Bologna and Paris honored him with their D.Sc.

In an expository style which is lucid, spare and comfortable, Alexander left a work marked by ideas and methods which have borne rich mathematical fruit.

\section{BIBLIOGRAPHY}

1. O. Veblen and J. W. Alexander, Manifolds of $n$ dimensions, Ann. of Math. (2) 14 (1913), $163-178$.

2. - Manifolds of $n$ dimensions, Bull. Amer. Math. Soc. 19 (1912/13), 391.

3. J. W. Alexander, Proof of invariances of certain constants of analysis situs, Bull. Amer. Math. Soc. 20 (1913/14), 168.

4. - Sur les cycles des surfaces algébriques et sur une définition topologique de l'invariant de Zeuthen-Segre, Rom. Accad. Lincei Rend. 23 (1914), 55-62.

5. - A proof of the invariance of certain constants of analysis situs, Trans. Amer. Math. Soc. 16 (1915), 148-154.

6. - Normal forms for one- and two-sided surfaces, Ann. of Math. (2) 16 (1915), $158-161$.

7. - Functions which map the interior of the unit circle upon simple regions, Ann. of Math. (2) 17 (1915), 12-22.

8. - Some generalizations of the Jordan theorem, Bull. Amer. Math. Soc. 22 (1916), 492.

9. - On the factorization of Cremona plane transformations, Bull. Amer. Math. Soc. 22 (1915/16), 279; Trans. Math. Soc. 17 (1916), 295-300.

10. - Note on two three-dimensional manifolds with the same group, Trans. Amer. Math. Soc. 20 (1919), 339--342.

11. - Note on Riemann spaces, Bull. Amer. Math. Soc. 26 (1919/20), 370-372.

12. - A proof of Jordan's theorem about a simple closed curve, Ann. of Math. (2) 21 (1920), 180-184. 
13. O. Veblen and J. W. Alexander, On the equilibrium of a mass at rest, Trans. Amer. Math. Soc. 21 (1920), 446-450.

14. - On transformations with invariant points, Trans. Amer. Math. Soc. 23 (1922), $89-95$.

15. - Some theorems on transformations with invariant points, Bull. Amer. Math. Soc. 28 (1922), 10.

16. - Theorem on the interior of a simply connected closed surface in three-space, Bull. Amer. Math. Soc. 28 (1922), 10.

17. ——, A proof and extension of the Jordan-Brouwer separation theorem, Trans. Amer. Math. Soc. 23 (1922), 333-349.

18. - Invariant points of a surface transformation of given class, Trans. Amer. Math. Soc. 25 (1923), 173-184.

19. - A lemma on systems of knotted curves, Proc. Nat. Acad. Sci. U.S.A. 9 (1923), $93-95$.

20. - On the deformation of an n-cell, Proc. Nat. Acad. Sci. U.S.A. 9 (1923), 406-407.

21. - Invariant points of surface transformations, Bull. Amer. Math. Soc. 29 (1923), 116.

22. - The existence of closed geodesics on surfaces (Preliminary communication), Bull. Amer. Math. Soc. 29 (1923), 148-149.

23. - On the deformation of an $n$-cell, Bull. Amer. Math. Soc. 30 (1924), 10.

24. - On infinitely connected plane regions, Bull. Amer. Math. Soc. 30 (1924), 10.

25. - Note on Brouwer's fixed point theorem, Bull. Amer. Math. Soc. 30 (1924), 299-300.

26. - On the subdivision of 3-space by a polyhedron, Proc. Nat. Acad. Sci. U.S.A. 10 (1924), 6-8.

27. - An example of a simply connected surface bounding a region which is not simply connected, Proc. Nat. Acad. Sci. U.S.A. 10 (1924), 8-10.

28. - Remarks on a point set constructed by Antoine, Proc. Nat. Acad. Sci. U.S.A. 10 (1924), 10-12.

29. - New topological invariants expressible as tensors, Proc. Nat. Acad. Sci: U.S.A. 10 (1924), 99-101.

30. - On certain new topological invariants of a manifold, Proc. Nat. Acad. Sci. U.S.A. 10 (1924), 101-103.

31. - Topological invariants of manifolds, Proc. Nat. Acad. Sci. U.S.A. 10 (1924), 493-494.

32. - On the intersection invariants of a manifold, Proc. Nat. Acad. Sci. U.S.A. 11 (1925), 143-146.

33. - Note on a theorem by H. Kneser, Proc. Nat. Acad. Sci. U.S.A. 11 (1925), $250-251$.

34. - On the decomposition of tensors, Ann. of Math. (2) 27 (1925/26), 421-423.

35. - Combinatorial analysis situs, Trans. Amer. Math. Soc. 28 (1926), 301-329.

36. - On the class of a covariant tensor, Ann. of Math. (2) 28 (1926/27), 245-250.

37. J. W. Alexander and G. B. Briggs, On types of knotted curves, Ann. of Math. (2) 28 (1926/1927), 562-586.

38. J. W. Alexander, Topological invariants of knotted curves in 3-space, Bull. Amer. Math. Soc. 33 (1927), 412.

39. - Topological invariants of knots and links, Trans. Amer. Math. Soc. 30 (1928), $275-306$.

40. - The combinatorial theory of complexes, Ann. of Math. (2) 31 (1930), 292-320. 
41. J. W. Alexander and L. W. Cohen, Homology groups associated with infinite complexes, Bull. Amer. Math. Soc. 37 (1931), 345.

42. - A classification of the homology groups of compact spaces, Ann. of Math. (2) 33 (1932), 538-566.

43. J. W. Alexander, Some problems in topology, Verhandlungen Kongress Zurich 1932, 249-257.

44. - - A matrix knot invariant, Proc. Nat. Acad. Sci. U.S.A. 19 (1933), 272-275.

45. - On the homology groups of abstract spaces, Ann. of Math. (2) 35 (1934), 130151.

46. - On the characters of discrete abelian groups, Ann. of Math. (2) 35 (1934), $389-395$.

47. - Note on Pontrjagin's topological theorem of duality, Proc. Nat. Acad. Sci. U.S.A. 21 (1935), 222-225.

48. - On the chains of a complex and their duals, Proc. Nat. Acad. Sci. U.S.A. 21 (1935), 509-511.

49. - On the ring of a compact metric space, Proc. Nat. Acad. Sci. U.S.A. 21 (1935), $511-512$.

50. - On non-periodic forces, acting on oscillatory systems, Physica 2 (1935), 273-285.

51. J. W. Alexander and L. Zippin, Discrete abelian groups and their character groups, Ann. of Math. (2) 36 (1935), 71-85.

52. J. W. Alexander, On the connectivity ring of an abstract space, Ann. of Math. (2) 37 (1936), 698-708.

53. - On the connectivity ring of a bicompact space. I, II, Proc. Nat. Acad. Sci. U.S.A. 22 (1936), 300-303, 381-384.

54. - A theory of connectivity in terms of gratings, Ann. of Math. (2) 39 (1938), $883-912$

55. - On the concept of a topological space, Proc. Nat. Acad. Sci. U.S.A. 25 (1939), $52-54$.

56. - On the connectivity ring of a lattice, Proc. Nat. Acad. Sci. U.S.A. 25 (1939), $208-209$

57. - Ordered sets, complexes and the problems of compactification, Proc. Nat. Acad. Sci. U.S.A. 25 (1939), 296-298.

58. - Gratings and homology theory, Bull. Amer. Math. Soc. 53 (1947), 201-233. MR 8, 480 .

59. - The connectivity ring of an abstract space, Uspehi Mat. Nauk 2 (1947), no. 1(17), 156-165. (Russian) MR 10, 317. 